\title{
Dynamic Analysis of Total Factor Productivity (TFP) of Traditional Chinese Medicine (TCM) Hospitals in Hubei Province from 2013 to 2019
}

\author{
TANG Cheng ${ }^{1}$, MA Jun ${ }^{1}$, CHEN Li ${ }^{* 1}$ \\ ${ }^{1}$ School of Management, Hubei University of Chinese Medicine, Wuhan, 430065
}

\begin{abstract}
Objectives: To study the changes of TFP in TCM hospitals in Hubei Province from 2013 to 2019, so as to provide policy basis for improving service efficiency and promoting the development of TCM. Methods: The statistical reports of 90 public TCM hospitals in Hubei Province from 2013 to 2019 were collected using the Hubei Provincial Comprehensive Statistical Information System for TCM, and the TFP of TCM hospitals were dynamically analysed using the DEA-Malmquist index method. Results: From 2013 to 2019, the Total Factor Productivity Change (TFPCH) index of TCM Hospitals in Hubei Province increased by $0.8 \%$; the Efficiency Change (EFFCH) index increased by $0.5 \%$; the Technical Change (TECH) index increased by $0.3 \%$; the Pure Efficiency Change (PECH) index increased by $0.2 \%$ and the Scale Efficiency Change (SECH) index increased by $0.4 \%$. The mean values of the TFPCH index in the eastern, central, and western regions of Hubei Province were 1.001, 1.011, and 1.013, respectively, and the mean values of that in the secondary and tertiary hospitals were 1.004 and 1.024, respectively. Conclusions: The TFP of TCM hospitals in Hubei Province tends to improve on the whole. There are differences in the TFPCH index of TCM hospitals in different regions and levels. The stage development characteristics of core cities play a leading role and are worth learning.
\end{abstract}

\section{Introduction}

Hubei Province has a unique advantage in the development of TCM due to its abundant TCM resources and time-honored TCM culture. In recent years, the government of Hubei Province has issued a series of policy documents, such as the Implementation Opinions on Comprehensively Promoting the Development of Traditional Chinese Medicine (Hubei Gov., [2016] Reference No. 81) and the Three-year Action Plan for Promoting the Construction of a Strong Province of Traditional Chinese Medicine (Hubei Gov., [2020] Reference No. 26), to actively promote the development of the TCM in Hubei Province and the construction of Hubei province as a strong province of TCM. Public TCM hospitals are an important part of TCM service system, and their service capabilities and service efficiency can affect the future development of TCM. In this study, we analyzed the TFP of 90 Public TCM hospitals in Hubei Province from 2013 to 2019 based on DEA-Malmquist index method to understand its dynamic changes and provide data support and policy suggestions for improving hospital service efficiency.

\section{Source and Research Methodology}

\subsection{Source}

\footnotetext{
* Corresponding author:Chen Li, chenli0401@163.com
}

The statistical reports of Public TCM Hospitals in Hubei Province from 2013 to 2019 were collected using the Hubei Provincial Comprehensive Statistical Information System for TCM. Excluding the newly built hospitals after 2013, a total of 90 TCM hospitals were included in this study, including 67 secondary hospitals and 23 tertiary hospitals.

According to geographical location, the eastern part of Hubei Province includes Huangshi, Huanggang and Xianning; the central part includes Wuhan, Tianmen, Xiantao, Qianjiang, Jingmen, Xiaogan, Suizhou, and E'zhou; the western part includes Shiyan, Yichang, Xiangyang, Jingzhou, Enshi, Shennongjia Forestry District $^{[1]}$. There are 20, 29 and 41 TCM hospitals in the eastern, central and western regions, respectively.

\subsection{Research Methodology}

\subsubsection{DEA-Malmquist index method}

The Malmquist index was first proposed by Malmquist ${ }^{[2]}$ in the 1950s. After continuous development, on the basis of the theory by Fare ${ }^{[3]}$ et al in 1992-DEA theory ${ }^{[4]}$ and combined with Malmquist TFP index established by Caves $^{[5]}$ et al., a DEA-Malmquist index model was constructed to explore the changes of TFP index of Decision Making Units (DMU) with multi-input and multi-output during continuous periods. To judge the 
main factors of TFP, the TFP index can be decomposed into TECH and EFFCH. The EFFCH index can be further decomposed into $\mathrm{PECH}$ and $\mathrm{SECH}^{[6]}$. An index greater than 1 indicates that the efficiency of $\mathrm{T}+1$ phase is improved compared with $\mathrm{T}$ phase; an index equal to 1 indicates that the efficiency of $\mathrm{T}+1$ phase is not changed compared with $\mathrm{T}$ phase; and an index less than 1 indicates that the efficiency of $\mathrm{T}+1$ phase is decreased compared with $\mathrm{T}$ phase ${ }^{[7]}$.

\subsubsection{Statistical analysis}

The statistical data of TCM Hospitals in Hubei province from 2013 to 2019 were input by Excel and were analyzed by the method of descriptive statistics. DEAMalmquist analysis was performed using DEAP 2.1.

\subsection{Selection of indicators}

The similar research literatures are summarized ${ }^{[8-11]}$. Combined with the existing data, four output indicators are selected, including the number of diagnosis and treatment patients, the number of discharged patients, the bed utilization rate and medical income; three input indicators are selected, including the number of available beds, the number of fully employed staff and the house construction area. Individual missing data were imputed by calculating the arithmetic mean from the pre and post 2-year data.

\section{Results}

\subsection{Input and output indicators of TCM Hospital in Hubei Province from 2013 to 2019}

Table 1. Analysis of input and output indicators of TCM Hospitals in Hubei Province from 2013 to 2019

\begin{tabular}{|l|l|l|r|r|r|c|c|}
\hline \multirow{2}{*}{ Year } & \multicolumn{4}{|c|}{ Output indicators } & \multicolumn{4}{c|}{ Input indicators } \\
\cline { 2 - 9 } & $\begin{array}{l}\text { Total number } \\
\text { of patients }\end{array}$ & $\begin{array}{l}\text { Number of } \\
\text { discharges }\end{array}$ & $\begin{array}{l}\text { Bed } \\
\text { utilization } \\
\text { rate /\% }\end{array}$ & $\begin{array}{l}\text { Medical } \\
\text { income/10,00 } \\
\text { 0 yuan }\end{array}$ & $\begin{array}{l}\text { Number of } \\
\text { available beds }\end{array}$ & $\begin{array}{l}\text { Number } \\
\text { employed } \\
\text { staff }\end{array}$ & $\begin{array}{l}\text { Hospital building } \\
\text { area/m }{ }^{2}\end{array}$ \\
\hline 2013 & 17875851 & 1030810 & 92.80 & 827530 & 29633 & 33089 & 2198827 \\
\hline 2014 & 19462922 & 1136158 & 92.31 & 985568 & 32216 & 36698 & 2386002 \\
\hline 2015 & 20121970 & 1201473 & 88.31 & 1039030 & 36407 & 39168 & 2550965 \\
\hline 2016 & 20636861 & 1266583 & 87.68 & 1127138 & 38461 & 40826 & 3109750 \\
\hline 2017 & 22177849 & 1376844 & 88.51 & 1231962 & 40561 & 42051 & 3382360 \\
\hline 2018 & 22486909 & 1422285 & 88.79 & 1374983 & 42177 & 43275 & 3394633 \\
\hline 2019 & 23063218 & 1506178 & 92.58 & 1550399 & 43173 & 43825 & 3547512 \\
\hline
\end{tabular}

\subsection{Analysis of TFPCH index and decomposition index of TCM Hospitals in Hubei Province from the perspective of time series}

Table 2 shows the TFPCH index and the decomposition of Malmquist index of TCM Hospitals in Hubei Province from 2013 to 2019 . It can be seen that the mean values of each index were greater than 1 , showing an overall trend of optimization. The TFPCH index increased by
In terms of output indicators, the annual average growth rate of total patients from 2013 to 2019 was $4.34 \%$, including $3.01 \%, 5.68 \%$ and $3.09 \%$ in the eastern, central and western Hubei Province, respectively, and $4.28 \%$ and $4.38 \%$ in the secondary and tertiary TCM hospitals, respectively. The annual average growth rate of the number of discharges was $6.52 \%$, including $8.06 \%$, $7.83 \%$, and $4.66 \%$ in the eastern, central and western Hubei Province, respectively, and $8.06 \%$ and $4.66 \%$ in the secondary and tertiary hospitals, respectively. The bed utilization rate was maintained at around $90 \%$, with a small decrease in 2015-2018. The annual average increase in medical income was $11.03 \%$, including $12.59 \%, 10.96 \%$, and $10.53 \%$ in the eastern, central and western Hubei Province, respectively, and $12.75 \%$ and $9.92 \%$ in the secondary and tertiary hospitals. In terms of input indicators, the annual average growth rate of the number of available beds was $6.47 \%$, including $6.76 \%$, $8.29 \%$, and $4.81 \%$ in the eastern, central, and western Hubei Province, respectively, and $7.93 \%$ and $4.71 \%$ in the secondary and tertiary hospitals. The annual average growth rate of fully employed staff was $4.79 \%$, including $4.25 \%, 5.71 \%$, and $4.16 \%$ in the eastern, central, and western Hubei Province, respectively, and 5.47\% and $4.05 \%$ in the secondary and tertiary hospitals. The annual average growth rate of house construction area was $8.30 \%$, including $11.90 \%, 12.68 \%$, and $2.09 \%$ in the eastern, central, and western Hubei Province, respectively, and $10.42 \%$ and $5.78 \%$ in the secondary and tertiary hospitals. In general, the growth rate of input and output indicators in the secondary hospitals was higher than that in the tertiary hospitals, the growth rate of input indicators in central regions was higher than that in eastern and western regions, and the growth rate of output indicators in eastern regions was slightly higher than that in central and western regions. As shown in Table 1 .

$0.8 \%$ per year. Except for a decrease of $9.3 \%$ in $2014-$ 2015 , it increased in all other years, with a large increase in 2013 - 2014 and 2018 - 2019 (both more than 5\%). The average annual increase of EFFCH index was $0.5 \%$, with the largest decrease of $8.6 \%$ in 2013 - 2014. It increased in all other years, with the largest increase of $4.1 \%$ in $2015-2016$. The average annual increase of TECH index was $0.3 \%$, which decreased in 2014-2017, with the maximum decrease of $11.3 \%$ (2014-2015) and the maximum increase of $16.7 \%$ in $2013-2014$. The 
average PECH index increased by $0.2 \%$ per year, with the largest increase of $4.3 \%$ in 2016 - 2017 and small fluctuations in other years. The average SECH index increased by $0.4 \%$ per year, with the largest increase of $2.9 \%$ in $2018-2019$.

Table 2. TFPCH and decomposition of Malmquist index of TCM Hospitals in Hubei Province from 2013 to 2019

\begin{tabular}{cccccc}
\hline Year & EFFCH & TECH & PECH & SECH & TFPCH \\
\hline $2013-2014$ & 0.914 & 1.167 & 0.952 & 0.960 & 1.067 \\
$2014-2015$ & 1.023 & 0.887 & 1.000 & 1.023 & 0.907 \\
$2015-2016$ & 1.041 & 0.966 & 1.016 & 1.024 & 1.005 \\
$2016-2017$ & 1.038 & 0.972 & 1.043 & 0.995 & 1.008 \\
$2017-2018$ & 1.005 & 1.013 & 1.013 & 0.992 & 1.018 \\
$2018-2019$ & 1.016 & 1.034 & 0.987 & 1.029 & 1.050 \\
Mean value & 1.005 & 1.003 & 1.002 & 1.004 & 1.008 \\
\hline
\end{tabular}

\subsection{Analysis of TFPCH index and decomposition index of TCM Hospitals in Hubei Province from the geographical area dimension}

The TFPCH index and decomposition of Malmquist index of TCM hospitals in Hubei Province from 2013 to 2019 are shown in Table 3. The average values of TFPCH index in eastern, central and western Hubei Province were $1.001,1.011$ and 1.013 , respectively. Efficiency has been improved in all areas, especially in the western part of Hubei Province. According to the analysis of hospitals at different levels, the mean values of TFPCH index in secondary and tertiary hospitals were 1.004 and 1.024, respectively, and the efficiency improvement in tertiary hospitals was better than that in secondary hospitals.

Among the seventeen prefectures and cities in Hubei Province, nine achieved the improvement of TFP, of which the average TFPCH index in Shennongjia Forestry District increased by $11.6 \%$ per year, with the most significant improvement in efficiency. Of these nine prefectures and cities, seven are driven by both technological progress and technological efficiency improvement to achieve improvement of TFP. Further analysis of the EFFCH index revealed that the above seven prefectures and cities achieved improvement in Pure Efficiency, and three (Enshi Prefecture, Xiangyang City, and E'zhou City) achieved improvement in Scale Efficiency.

According to the analysis of eight prefectures and cities that did not achieve the improvement of TFP, the reduction of TFP in Tianmen City and Jingzhou City was caused by both technical regression and technical efficiency reduction, the reduction of TFP in Yichang City and Qianjiang City were caused by the reduction of technical efficiency, and the reduction of TFP in Huanggang City, Shiyan City, Xiaogan City and Xianning City was caused by technical regression.

Table 3. TFPCH and decomposition of Malmquist index of TCM Hospitals in Hubei Province from 2013 to 2019

\begin{tabular}{|c|c|c|c|c|c|}
\hline & EFFCH & TECH & PECH & SECH & TFPCH \\
\hline Wuhan City & 1.003 & 1.007 & 1.005 & 0.998 & 1.010 \\
\hline Huangshi City & 1.027 & 1.011 & 1.027 & 1.000 & 1.038 \\
\hline Shiyan City & 1.012 & 0.986 & 1.002 & 1.010 & 0.997 \\
\hline Xiangyang City & 1.010 & 1.020 & 1.007 & 1.003 & 1.032 \\
\hline Yichang City & 0.983 & 1.004 & 0.978 & 1.005 & 0.987 \\
\hline Jingzhou City & 0.989 & 0.996 & 0.987 & 1.002 & 0.986 \\
\hline Jingmen City & 1.006 & 1.023 & 1.007 & 0.999 & 1.029 \\
\hline E'zhou City & 1.059 & 1.012 & 1.058 & 1.001 & 1.072 \\
\hline Xiaogan City & 1.003 & 0.995 & 1.004 & 0.998 & 0.998 \\
\hline Huanggang City & 1.004 & 0.988 & 0.999 & 1.005 & 0.992 \\
\hline Xianning City & 1.004 & 0.995 & 1.004 & 1.001 & 0.999 \\
\hline Suizhou City & 0.995 & 1.010 & 0.996 & 1.000 & 1.005 \\
\hline Enshi Prefecture & 1.023 & 1.025 & 1.021 & 1.002 & 1.048 \\
\hline Xiantao City & 1.028 & 1.035 & 1.028 & 0.999 & 1.063 \\
\hline Tianmen City & 0.980 & 0.995 & 0.959 & 1.022 & 0.976 \\
\hline Qianjiang City & 0.971 & 1.018 & 0.971 & 1.000 & 0.988 \\
\hline $\begin{array}{l}\text { Shennongjia Forestry } \\
\text { District }\end{array}$ & 1.169 & 0.955 & 1.062 & 1.101 & 1.116 \\
\hline
\end{tabular}

\subsection{Analysis of TFPCH index and decomposition index of TCM Hospitals in core cities of Hubei Province}

According to the strategic layout of the Yangtze River Economic Belt, the development trend of Wuhan, Xiangyang and Yichang, as the "one body" and "two wings" core cities in Hubei section, has an important leading and exemplary role for other cities in the same region. The efficiency analysis of these cities is helpful to provide reference for the development of TCM hospitals in urban agglomeration areas of "Wuhan $1+8$ urban circle", "Xiangyang-Shiyan-Suizhou-Shennongjia" and "Yichang-Jingzhou-Jingmen-Enshi". The TFPCH index and decomposition of Malmquist index of 
different levels of TCM hospitals in the core area are shown in Table 4.

Table 4. TFPCH and Decomposition of Malmquist index of TCM hospitals in core cities of Hubei Province from 2013 to 2019

\begin{tabular}{llccccc}
\hline & & EFFCH & TECH & PECH & SECH & TFPCH \\
\hline \multirow{2}{*}{ Wuhan City } & Tertiary & 0.991 & 1.007 & 0.993 & 0.999 & 0.997 \\
& Secondary & 1.011 & 1.008 & 1.013 & 0.998 & 1.019 \\
Xiangyang & Tertiary & 1.002 & 1.023 & 1.002 & 1.000 & 1.025 \\
City & Secondary & 1.011 & 1.020 & 1.007 & 1.004 & 1.033 \\
Yichang & Tertiary & 1.002 & 1.007 & 0.991 & 1.010 & 1.009 \\
City & Secondary & 0.981 & 1.004 & 0.977 & 1.004 & 0.984 \\
\hline
\end{tabular}

From the above table, it can be seen that the TFPCH index of other TCM hospitals have been increased except tertiary TCM hospitals in Wuhan and secondary TCM hospitals in Yichang. The decomposition of the index revealed that the TECH index of TCM hospitals at all levels in the three regions were greater than 1, and the decrease of the EFFCH index was the reason for the decrease of the TFPCH index of tertiary TCM hospitals in Wuhan and secondary TCM hospitals in Yichang. Further decomposition of the technical efficiency revealed that the PECH index of tertiary TCM hospitals in Wuhan decreased by $0.7 \%$; the SECH index decreased by $0.1 \%$, and the PECH index of secondary TCM hospitals in Yichang decreased by $2.3 \%$. The TFPCH index of secondary and tertiary TCM hospitals in Xiangyang increased by $3.3 \%$ and $2.5 \%$, respectively, of which technical progress was the main engine, and the TECH index increased by $2.0 \%$ and $2.3 \%$, respectively.

\section{Discussions and suggestions}

\subsection{The TFP of TCM hospitals in Hubei Province tended to improve on the whole}

The mean TFPCH index of TCM hospitals in Hubei Province from 2013 to 2019 was 1.008 , increased by $0.8 \%$. In terms of the decomposition of the index, the EFFCH index increased by $0.5 \%$ and the TECH index increased by $0.3 \%$. Further decomposition of the EFFCH index showed that the PECH index increased by $0.2 \%$ and the SECH index increased by $0.4 \%$. It can be seen that under the combined effect of improving pure technical efficiency, scale efficiency, and technical progress, the efficiency of TCM hospitals in Hubei Province tended to improve on the whole. It benefited from the promulgation and implementation of a series of policies in Hubei Province, such as the Decision on Accelerating the Development of Traditional Chinese Medicine, the Implementation Opinions on Comprehensively Promoting the Development of Traditional Chinese Medicine, and the Three-year Action Plan of Hubei Province for Promoting the Construction of a Strong Province of Traditional Chinese Medicine. As a result, the development of TCM hospitals has ushered in new opportunities. At present, all TCM hospitals in Hubei Province should take the 14th Five Year Plan as an opportunity to integrate the development of TCM hospitals into the strategic layout of all cities and prefectures, so as to promote the sustainable development of TCM hospitals.

\subsection{There are differences in TFPCH index among different regions and different levels of TCM hospitals}

From 2013 to 2019 , the mean values of TFPCH index were $1.001,1.011$, and 1.013 in eastern, central, and western Hubei provinces, respectively, 1.004 and 1.024 in secondary and tertiary hospitals, respectively, and $1.005,1.018,1.010$ in "Wuhan $1+8$ urban circle", "Xiangyang-Shiyan-Suizhou-Shennongjia" and "Yichang-Jingzhou-Jingmen-Enshi", respectively. It can be seen that there were differences in the TFPCH index of different regions and different levels of TCM hospitals. Further analysis revealed that the TECH index decreased by $0.6 \%$ in the eastern region, $0.1 \%$ in the secondary hospitals, $0.1 \%$ in the "Wuhan $1+8$ urban circle", and the EFFCH index decreased by $0.1 \%$ in the " Yichang-Jingzhou-Jingmen-Enshi", of which the PECH index decreased by $0.3 \%$, and the existing problems in hospitals at all levels were not the same. For the TCM hospitals with declining TECH index, with the development of the times, the technological progress did not show a trend of synchronous growth with other production factors, showing a regressive state relative to other production factors. For areas where the $\mathrm{PECH}$ index decreased, it is necessary to optimize the structure of input factors and output factors, avoid the problems of input redundancy and insufficient output, and promote the improvement of technical efficiency. In addition, as a whole, Hubei Province can establish a peer-to-peer assistance mechanism among various regions and build a "medical treatment alliance" among urban agglomeration in combination with the actual situation in the context of the policy of building a strong province of TCM, so as to explore the potential for technical progress, narrow the development gap of TCM hospitals in different regions and realize common development.

\subsection{The characteristics of stage development of core cities have a leading role and reference significance}

It is important for the development of TCM hospitals in non-core cities to actively visit and study the development mode of core cities and draw on their advanced experience combining with local situation. TCM hospitals in Wuhan, Xiangyang and Yichang can 
continuously export advanced management modes to surrounding areas and share the digital dividends of health care reform to strengthen medical cooperation and exchange. Hubei Province should promote the coconstruction and sharing of medical achievements and interconnect the services of "cloud hospitals". A joint development model with complementary advantages and all-region coordination should be formed through exchanges and cooperation, so as to realize the point-toarea strategy and enhance the radiation and leading role of core cities.

\section{Conclusion}

From 2013 to 2019, the TFP of TCM hospitals in Hubei Province has been improved as a whole, but there are still some differences among different regions and hospitals at all levels. TCM hospitals still need to continue to explore, optimize the input and output structure of health resources, strengthen hospital information construction, promote high-level TCM talent training, strengthen skills training for in-service personnel, explore suitable development scales, and continuously improve the service efficiency of TCM hospitals in Hubei Province.

\section{Acknowledgments}

Fund Project: The Project of Consultant and Research of Hubei Research Institute of China Engineering Science and Technology Development Strategy in 2020: Research on the Development Strategy of Traditional Chinese Medicine and the Massive Health Industry in Hubei Province.

\section{References}

1. Ye Chu-sheng, Xia Shan-hua. On Coordinative Development of Hubei Regional Economy [J]. Journal of Hubei University of Economics, 2005(04):87-92. (In Chinese)

2. Malmquist S. Index Numbers and Indifference Curves[J]. Trabajos de Estatistica, 1953,4(1):209242.

3. Fare R, Grosskopf S, Lindgren B, etal. Productivity Changes in Swedish Pharamacies 1980-1989: A non-parametric Malmquist Approach[J]. Journal of Productivity Analysis, 1992, 3(1):81-97.

4. Charnes A, Cooper W W, Rhodes E. Measuring the Efficiency of Decision Making Units. European Journal of Operations Research.1978,2(6):429-445.

5. Caves D W,Christensen L R, Diewert W E. The Economic Theory of Index: Numbers and the Measurement of Input, Output, and Productivity[J]. Econometrica,1982,50(6):1393-1414.

6. Wang Min, Li Ji-wei, Luo Cun, et al. The Danamic Analysis on the Total Factor Productivity of Hospitals of Traditional Chinese Medicine from
2002 to 2012 in Beijing J. Chinese Health Economics, 2013,32(12):78-80. (In Chinese)

7. Yang Xi, Chen Man-li. Trend of Total Factor Productivity of Township Health Services in China during 2003-2014 [J]. Chinese General Practice, 2018, 21(2):88-92. (In Chinese)

8. Dong Si-ping, Zuo Yu-ling, Tao Hong-bing, etc. Study on DEA-based Chinese Hospital Efficiency and Applied Indicators [J]. Chinese Journal of Health Policy, 2014, 7(010):40-45. (In Chinese)

9. Li Jing, Chen You-xian, Zhang Xiao-qin. DEA Analysis on Efficiency of Health Resource Allocation between Public Hospitals of Traditional Chinese Medicine and Private Hospitals of Traditional Chinese Medicine [J]. Chinese Journal of Health Statistics, 2020,037(001):80-82. (In Chinese)

10. Zhang Chao, Wang Xiao-jie. Analysis of Operation Efficiency of County-Level Hospitals of Traditional Chinese Medicine in Heilongjiang Province Based on DEA Model [J]. Medicine and Society, 2019, 032(003):87-90. (In Chinese)

11. Huang He-chong, Li Dong-sheng. Efficiency Evaluation of DEA Model Based on VRS-C2R for 16 Hospitals of Traditional Chinese Medicine in Guangdong Province [J]. Chinese Journal of Health Statistics, 2015, 32(003):500-501. (In Chinese) 